\title{
Effectiveness and safety of noninvasive positive pressure ventilation in the treatment of COVID-19-associated acute hypoxemic respiratory failure: a single center, non-ICU setting experience
}

\author{
Antonio Faraone ${ }^{1}$ (1) $\cdot$ Chiara Beltrame $^{1} \cdot$ Andrea Crociani $^{1} \cdot$ Paolo Carrai $^{1}$ - Elena Lovicu ${ }^{1}$. Simona Filetti ${ }^{1}$. \\ Serena Sbaragli ${ }^{1}$. Chiara Alessi ${ }^{1}$ - Michela Cameron Smith ${ }^{1}$. Chiara Angotti ${ }^{1}$. Alberto Fortini ${ }^{1}$
}

Received: 1 September 2020 / Accepted: 31 October 2020 / Published online: 22 November 2020

(c) Società Italiana di Medicina Interna (SIMI) 2020

\begin{abstract}
The role of noninvasive positive pressure ventilation (NIPPV) in COVID-19 patients with acute hypoxemic respiratory failure (AHRF) is uncertain, as no direct evidence exists to support NIPPV use in such patients. We retrospectively assessed the effectiveness and safety of NIPPV in a cohort of COVID-19 patients consecutively admitted to the COVID-19 general wards of a medium-size Italian hospital, from March 6 to May 7, 2020. Healthcare workers (HCWs) caring for COVID-19 patients were monitored, undergoing nasopharyngeal swab for SARS-CoV-2 in case of onset of COVID-19 symptoms, and periodic SARS-CoV-2 screening serology. Overall, 50 patients (mean age 74.6 years) received NIPPV, of which 22 (44\%) were successfully weaned, avoiding endotracheal intubation (ETI) and AHRF-related death. Due to limited life expectancy, $25(50 \%)$ of 50 NIPPV-treated patients received a "do not intubate" (DNI) order. Among these, only $6(24 \%)$ were weaned from NIPPV. Of the remaining 25 NIPPV-treated patients without treatment limitations, 16 (64\%) were successfully weaned, $9(36 \%)$ underwent delayed ETI and, of these, $3(33.3 \%)$ died. NIPPV success was predicted by the use of corticosteroids (OR 15.4, CI 1.79-132.57, $p$ 0.013) and the increase in the $\mathrm{PaO}_{2} / \mathrm{FiO}_{2}$ ratio measured 24-48 $\mathrm{h}$ after NIPPV initiation (OR 1.02 , CI $1-1.03, p 0.015$ ), while it was inversely correlated with the presence of a DNI order (OR 0.03, CI $0.001-0.57, p$ 0.020). During the study period, 2 of $124(1.6 \%) \mathrm{HCWs}$ caring for COVID-19 patients were diagnosed with SARS-CoV-2 infection. Apart from patients with limited life expectancy, NIPPV was effective in a substantially high percentage of patients with COVID-19-associated AHRF. The risk of SARS-CoV-2 infection among HCWs was low.
\end{abstract}

Keywords COVID-19 Respiratory distress syndrome $\cdot$ adult $\cdot$ Noninvasive ventilation $\cdot$ Aerosol-generating procedures Health personnel $\cdot$ Personal protective equipment

\section{Introduction}

Within a span of months, the COVID-19 pandemic, caused by severe acute respiratory syndrome coronavirus 2 (SARSCoV-2), has seen a surge of patients with respiratory distress admitted to hospitals across the globe [1]. Patients presenting with COVID-19-associated acute hypoxemic respiratory failure (AHRF) despite conventional oxygen therapy commonly meet the criteria for acute respiratory distress syndrome (ARDS) and require ventilatory support. Across

Antonio Faraone

antonio.faraone@uslcentro.toscana.it; antfaraone@gmail.com

1 Department of Internal Medicine, San Giovanni Di Dio Hospital, Via di Torregalli 3, 50143 Florence, Italy the globe, $7-26 \%$ of patients hospitalized with COVID-19 had critical illness with AHRF and were managed in the intensive care unit (ICU) setting, in most cases by invasive mechanical ventilation (MV) [2]. The role of noninvasive positive pressure ventilation (NIPPV) in patients with COVID-19-related AHRF is uncertain, as there is no direct evidence to support NIPPV in such patients [3, 4]. Studies on the use of NIPPV in AHRF with an etiology other than cardiogenic pulmonary edema have shown high rates of failure, with endotracheal intubation (ETI) rates up to $50 \%$ and high mortality [5-7]. Furthermore, there is concern that NIPPV use might increase the risk of nosocomial transmission of SARS-CoV-2 to healthcare workers (HCWs) by generating aerosols of infectious particles [4, 8-11].

Reflecting the current knowledge gap regarding risks and benefits associated with NIPPV use, international guidelines 
on clinical management of COVID-19 make only weak, conditional recommendations in favor of NIPPV, advocating the use of non-invasive ventilation in selected patients with mild ARDS, under close monitoring for worsening of respiratory failure, provided that appropriate airborne precautions are implemented [3, 4, 12].

The purpose of this study was to assess the effectiveness of NIPPV in treating AHRF in COVID-19 patients admitted to non-ICU wards and to explore predictors of NIPPV success. Our secondary objective was to evaluate the risk of SARS-CoV-2 transmission among HCWs caring for NIPPVtreated COVID-19 patients.

\section{Methods}

\section{Study design and participants}

We conducted a single-center retrospective analysis of patients with COVID-19-associated AHRF who were consecutively admitted to the COVID-19 general wards of our institution between March 6 and May 7, 2020, during the initial surge of the COVID-19 epidemic in Italy. The diagnosis of SARS-CoV-2 infection was confirmed using a real-time reverse transcriptase-polymerase chain reaction (RT-PCR) assay on nasopharyngeal swab specimens. All patients with COVID-19-associated AHRF who underwent NIPPV for at least a $2 \mathrm{~h}$ trial were recruited. In addition, we evaluated all HCWs who were in charge of the COVID-19 wards during the study period, exploring the risk of nosocomial SARS$\mathrm{CoV}-2$ infection. The institutional review board approved the study. Informed consent from patients was not required due to the observational nature of the study; verbal informed consent was acquired from HCWs.

\section{Clinical setting}

The study was performed in the 3 COVID-19 general wards of the San Giovanni di Dio Hospital, a medium-size acute care hospital (296 beds) in Florence, Italy. The COVID-19 general wards were equipped with 11 single, 8 double and 8 quadruple rooms, for a total of 59 beds. Patients were assigned to single rooms or cohorted in multiple-bed rooms, depending on availability. The wards had a closed ventilation system with air recirculation; no negative pressure rooms or high-efficiency particulate air (HEPA) filtering systems were available. Transition zones for donning and doffing of protective personal equipment (PPE) were created at the entry and exit of each ward, respectively. The wards were equipped with NIPPV ventilators and vital sign monitors for the assistance of severely to critically ill patients.

\section{COVID-19 wards staff}

The COVID-19 wards were managed by an exclusively dedicated internal medicine staff with the collaboration of some specialist doctors and surgeons. Overall, the staff was composed of a total of $124 \mathrm{HCWs}$, including 22 physicians, 61 nurses, 28 health assistants, and 13 ward assistants (HCWs in charge of cleaning the wards). All the involved healthcare personnel were trained to correctly use PPE. The medical staff was trained on the therapeutic management of COVID-19 patients and strictly collaborated with the intensive care consultants for the management of patients with respiratory failure.

During the period of activity, HCWs were closely monitored for the appearance of symptoms of SARS-CoV-2 infection; in addition, they underwent voluntary testing for SARS-CoV-2 serology twice, after the first month of activity and 2 weeks after the termination of activity in the COVID-19 wards. A two-tiered serologic testing algorithm was adopted, including an initial chromatographic rapid immunoassay, followed —in case of positive result—by a chemiluminescence quantitative immunoassay (iFlash1800 CLIA analyzer for anti-SARS-CoV-2 antibodies IgM and $\mathrm{IgG})$.

HCWs with COVID-19 symptoms or a positive serology test were subsequently evaluated by means of RT-PCR for SARS-CoV-2 on nasopharyngeal swab.

\section{Protective personal equipment}

HCWs attending the COVID-19 general wards were recommended to adopt airborne, droplet and contact precautions. PPE included a respirator mask (N95 respirators, FFP2, FFP3, or equivalent), a disposable long-sleeved gown or protective suit, double gloves, goggles or, in alternative, a face shield, shoe covers. Each HCW in charge of the COVID-19 general wards used a single piece of equipment (respirator, goggles or face shield, and gown or protective suit) for the entire work shift, in order to limit the consumption of PPE. Donning and doffing procedures were supervised by physician and nurse leaders. No remarkable shortage of PPE was registered at our COVID19 wards during the period of activity.

\section{NIPPV delivery}

NIPPV was delivered using a single-limb noninvasive ventilator (Philips Respironics V60), via a full-face or oronasal mask. In order to limit the production of virus-laden aerosols, nonvented masks were used, and a viral-bacterial 
filter was placed before the exhalation port of the NIPPV circuit.

The criteria for initiating NIPPV were: (a) a $\mathrm{SpO}_{2}<92 \%$ ( $<88 \%$ for patients with chronic obstructive pulmonary disease, COPD) despite conventional oxygen therapy; (b) respiratory fatigue; c) hypercapnia and respiratory acidosis.

Continuous positive airway pressure (CPAP) was the preferred mode of ventilation; positive end-expiratory pressure (PEEP) levels, along with the $\mathrm{FiO}_{2}$, were adjusted to achieve a $\mathrm{SpO}_{2}$ of $92-95 \%$ and a respiratory rate $\leq 25$ breaths $/ \mathrm{min}$, according to patient tolerance. Generally, a minimum PEEP of $5 \mathrm{cmH}_{2} \mathrm{O}$ was initially set, which was progressively incremented up to a maximum of $12 \mathrm{cmH}_{2} \mathrm{O}$, in order to achieve the oxygenation goal. A bilevel positive airway pressure (BiPAP) ventilation was applied to patients with COPD, hypercapnia, or persistent respiratory fatigue despite a brief trial of CPAP NIPPV. Inspiratory positive airway pressure (iPAP) was initially set at $15 \mathrm{cmH}_{2} \mathrm{O}$ and then adjusted, up to $20-25 \mathrm{cmH}_{2} \mathrm{O}$, to achieve respiratory rates of $\leq 25$ breaths/ min and exhaled tidal volumes of $6 \mathrm{~mL} / \mathrm{kg}$ predicted body weight. Expiratory positive airway pressure (ePAP) was adjusted to achieve target oxygenation. Vital signs were continuously monitored during NIPPV, and a blood gas analysis was performed with medical re-evaluation after the first hour of ventilation. Intensivist consultation and assessment for ETI were acquired for patients showing persistent or worsening respiratory distress after a $1 \mathrm{~h}$ NIPPV trial. Intubation was indicated if patients showed respiratory fatigue and/or failed to reach the oxygenation target. All patients achieving the oxygenation goal were maintained continuously on NIPPV for at least $6 \mathrm{~h}$ after initiation. Thereafter, the mask could be removed for short periods of up to $30 \mathrm{~min}$ each for meals and sputum clearance. When improvement in respiratory rate and decrease in oxygen requirement were evident, gradual weaning in the daytime followed by sleeping hours was performed. NIPPV was discontinued if the patient maintained a respiratory rate $\leq 25$ breaths $/ \mathrm{min}$ and a $\mathrm{SO}_{2} \geq 92 \%$ with a $\mathrm{FiO}_{2}$ of 0.5 without ventilatory support and activation of the accessory muscles of respiration.

\section{Study outcomes}

The primary outcome was the proportion of NIPPV-treated patients who were successfully weaned. Successful weaning was defined as the ability to be weaned from NIPPV for $\geq 24 \mathrm{~h}$. The need to switch to invasive MV and AHRFrelated death were considered NIPPV treatment failure.

Predictors of NIPPV success were explored among patient's demographic, clinical and treatment characteristics.

The secondary outcome was the percentage of SARS$\mathrm{CoV}-2$ infection acquisition among $\mathrm{HCW}$ s caring for COVID-19 patients. SARS-CoV-2 infection among HCWs was directly attributed to healthcare-associated transmission if (1) the infection was diagnosed within 14 days of termination of activity in the COVID-19 ward, and (2) the HCW had no other known exposure to SARS-CoV-2 infected individuals outside the COVID-19 ward.

\section{Data collection}

For each patient treated with NIPPV, the following demographic, clinical and treatment data were retrieved from electronic clinical records: sex, age, comorbidities, SOFA score, pre- and post-NIPPV arterial oxygen tension/inspiratory oxygen fraction $\left(\mathrm{PaO}_{2} / \mathrm{FiO}_{2}\right)$ ratio, NIPPV duration, modality of noninvasive ventilatory support (CPAP vs. BiPAP), drug treatments, and length of hospital stay. Patient outcomes (successful weaning from NIPPV, ETI for invasive ventilation, and in-hospital death) were also retrieved.

\section{Statistical analysis}

Continuous and categorical variables are presented as means (SD) and numbers (\%), respectively. Statistical analysis was performed using the Student's $t$ test for continuous variables and the Chi-square test or exact Fisher test for categorical variables, as appropriate. To explore the predictive factors associated with NIPPV success, univariable and multivariable logistic regression models were used. All data were analyzed using Med-Calc ${ }^{\circledR}$ version 12.3.0 (MedCalc Software; Mariakerke, Belgium). Statistical significance was set at $p<0.05$.

\section{Results}

During the study period a total of 143 patients were admitted to the 3 COVID-19 general wards of our institution. Fifty patients (33 males) were enrolled in the study, as they presented AHRF and underwent NIPPV. The mean age of NIPPV-treated patients was $74.6 \pm 11.0$ years, the mean number of comorbidities was $2.4 \pm 1.5$, the mean SOFA score was $3.1 \pm 1.2$ and the mean $\mathrm{PaO}_{2} / \mathrm{FiO}_{2}$ ratio before starting NIPPV was $130.1 \pm 63.5 \mathrm{mmHg}$. Due to advanced age, comorbidities and poor performance status, 25 (50\%) of the 50 NIPPV-treated patients had a limited life expectancy and received a treatment limitation decision, i.e. a "do not intubate" (DNI) order. The remaining 25 patients were deemed suitable for escalation to ETI and MV. Demographics, clinical characteristics, treatments, and outcomes of the study population are shown in Table 1 . All the enrolled patients received antithrombotic prophylaxis or treatment: $30(60 \%)$ were on low-molecular-weight-heparin (LMWH) prophylaxis, $8(16 \%)$ were on oral anticoagulant for atrial fibrillation and $12(24 \%)$ received LMWH at therapeutic doses for deep venous thrombosis or pulmonary embolism. Overall, 22 (44\%) of 50 patients responded to NIPPV and 
Table 1 Demographics, clinical characteristics, treatments, and outcomes among patients who were or were not weaned from NIPPV.

\begin{tabular}{|c|c|c|c|c|}
\hline Variables & Total & $\begin{array}{l}\text { Patients weaned from } \\
\text { NIPPV }\end{array}$ & $\begin{array}{l}\text { Patients not weaned } \\
\text { from NIPPV }\end{array}$ & $P$ value \\
\hline No & $50(100 \%)$ & $22(44 \%)$ & $28(56 \%)$ & \\
\hline Age, years, mean (SD) & $74.6(11)$ & $71(11.4)$ & $77.5(9.9)$ & 0.0361 \\
\hline Female, $n$ & $17(34 \%)$ & $6(27.3 \%)$ & $11(39.3 \%)$ & 0.5556 \\
\hline \multicolumn{5}{|l|}{ Comorbidities } \\
\hline Atrial fibrillation, $\mathrm{n}^{\circ}$ & $14(28 \%)$ & $5(22.7 \%)$ & $9(32.1 \%)$ & 0.6754 \\
\hline Cardiovascular disease, $n$ & $22(44 \%)$ & $8(36.4 \%)$ & $14(50 \%)$ & 0.4982 \\
\hline Chronic kidney disease, $n$ & $10(20 \%)$ & $2(9.1 \%)$ & $8(28.6 \%)$ & 0.1535 \\
\hline Cognitive impairment, $n$ & $7(14 \%)$ & $2(9.1 \%)$ & $5(17.9 \%)$ & 0.4444 \\
\hline $\mathrm{COPD}, n$ & $10(20 \%)$ & $4(18.2 \%)$ & $6(21.4 \%)$ & 1 \\
\hline Diabetes mellitus, $n$ & $12(24 \%)$ & $5(22.7 \%)$ & $7(25 \%)$ & 0.8518 \\
\hline Hypertension, $n$ & $28(56 \%)$ & $12(54.5 \%)$ & $16(57.1 \%)$ & 0.8543 \\
\hline Immunodeficiency, $n$ & $3(6 \%)$ & $2(9.1 \%)$ & $1(3.6 \%)$ & 0.5757 \\
\hline Malignancy, $n$ & $6(12 \%)$ & $1(4.5 \%)$ & $5(17.9 \%)$ & 0.2109 \\
\hline Stroke, $n$ & $8(16 \%)$ & $2(9.1 \%)$ & $6(21.4 \%)$ & 0.4391 \\
\hline Number of comorbidities, mean (SD) & $2.4(1.5)$ & $2(1.6)$ & $2.8(1.3)$ & 0.0569 \\
\hline SOFA score, mean (SD) & $3.1(1.2)$ & $2.9(1)$ & $3.3(1.4)$ & 0.2635 \\
\hline $\mathrm{PaO}_{2} / \mathrm{FiO}_{2}$ before NIPPV, mm Hg, mean (SD) & $130.1(63.5)$ & $136.8(57.9)$ & $124.9(68.2)$ & 0.5164 \\
\hline $\mathrm{PaO}_{2} / \mathrm{FiO}_{2} 24-48 \mathrm{~h}$ after NIPPV, mm Hg, mean (SD) & $148.3(63.5)$ & $183.2(64.7)$ & $120.8(70.2)$ & 0.0022 \\
\hline \multicolumn{5}{|l|}{ NIPPV modality } \\
\hline NIPPV BiPAP, $n$ & $25(50 \%)$ & $8(36.4 \%)$ & $17(60.7 \%)$ & 0.1543 \\
\hline NIPPV CPAP, $n$ & $25(50 \%)$ & $14(63.6 \%)$ & $11(39.3 \%)$ & 0.1543 \\
\hline NIPPV duration, hours, mean (SD) & $187(181)$ & $264(223.3)$ & $126.4(109.8)$ & 0.0063 \\
\hline \multicolumn{5}{|l|}{ Treatments } \\
\hline Treatment with corticosteroids, $n$ & $35(70 \%)$ & $19(86.4 \%)$ & $16(57.1 \%)$ & 0.0325 \\
\hline Treatment with tocilizumab, $n$ & $17(34 \%)$ & $12(54.5 \%)$ & $5(17.9 \%)$ & 0.0156 \\
\hline Treatment with antibiotics, $n$ & $46(92 \%)$ & $20(90.9 \%)$ & $26(92.9 \%)$ & 1 \\
\hline DNI order, $n$ & $25(50 \%)$ & $6(27.3 \%)$ & $19(67.9 \%)$ & 0.0103 \\
\hline \multicolumn{5}{|l|}{ Outcomes } \\
\hline Duration of hospital stay, days, mean (SD) & $19.6(14.5)$ & $23(11)$ & $15.3(13.1)$ & 0.0319 \\
\hline In-hospital death, $n$ & $25(50 \%)$ & $3(13.6 \%)$ & $22(78.6 \%)$ & $<0.0001$ \\
\hline
\end{tabular}

Table 2 Clinical features and outcomes of NIPPV-treated patients with or without a DNI order

\begin{tabular}{llll}
\hline Variables & $\begin{array}{l}\text { Patients with DNI } \\
\text { order }(n=25)\end{array}$ & $\begin{array}{l}\text { Patients without DNI } \\
\text { order }(n=25)\end{array}$ & $P$ value \\
\hline Age, years, mean (SD) & $82.1(7.6)$ & $67.1(8.4)$ & 0.0001 \\
Number of comorbidities, mean (SD) & $3(1.3)$ & $1.84(1.4)$ & 0.0039 \\
SOFA score, mean (SD) & $3.6(1.4)$ & $2.6(0.8)$ & 0.0032 \\
$\mathrm{PaO}_{2} / \mathrm{FiO} 2$ pre-NIPPV (SD) & $127.4(67.9)$ & $132.9(60.2)$ & 0.7632 \\
$\mathrm{PaO}_{2} / \mathrm{FiO}$ 2 post-NIPPV (SD) & $127(80.8)$ & $169.6(61.2)$ & 0.0409 \\
$\mathrm{NIPPV}_{2}$ success, $n$ & $6(24 \%)$ & $16(64 \%)$ & 0.0103 \\
Delayed ETI, $n$ & - & $9(36 \%)$ & - \\
In-hospital death, $n$ & $22(88 \%)$ & $3(12 \%)$ & $<0.0001$ \\
\hline
\end{tabular}

were successfully weaned. The success rate of NIPPV in patients with a DNI order was $24 \%(6 / 25)$ (Table 2). Among the 25 patients without treatment limitations, 16 (64\%) were weaned from NIPPV and subsequently discharged, and 9
(36\%) underwent delayed ETI and were transferred to the ICU due to worsening of respiratory distress (Table 2). For these patients, the mean time between NIPPV initiation and ETI was $55.3 \mathrm{~h} \pm 81.4$ (median $24 \mathrm{~h}$ ). Three (33.3\%) of the 
intubated patients died during the ICU stay; the remaining 6 were successfully weaned from MV and subsequently discharged from the hospital.

The overall in-hospital mortality among NIPPV-treated patients was 50\% (25/50) (Table 1). Mortality was significantly higher among patients with a treatment limitation decision than among patients with no preclusion to ETI ( $88 \%$ vs. $12 \%, p<0.0001$, Table 2 ).

In the multivariable analysis, the use of corticosteroids (OR 15.4, CI 1.79-132.57, $p$ 0.013), and the increase in the $\mathrm{PaO}_{2} / \mathrm{FiO}_{2}$ ratio measured $24-48 \mathrm{~h}$ after initiation of NIPPV (OR 1.02, CI 1-1.03, $p$ 0.015) were independently associated with NIPPV success, whereas the presence of a treatment limitation decision (OR 0.03, CI 0.001-0.57, $p$ 0.020 ), was a predictor of NIPPV failure (Table 3 ).

During the study period, 1 of $124 \mathrm{HCWs}$ (a health assistant) in charge of the 3 COVID-19 general wards developed mild symptoms (fatigue, myalgia, smell and taste loss, fever) and was diagnosed with SARS-CoV-2 infection by means of nasopharyngeal swab. All healthcare personnel underwent the 2 scheduled screening serology tests for SARS-CoV-2. The second test for SARS-CoV-2 antibodies was positive (IgG and $\mathrm{IgM}$ ) in 1 asymptomatic nurse, whose subsequent nasopharyngeal swab tested negative. Globally, based on the results of molecular and serologic assays, 1.6\% (2/124) of
HCWs experienced a healthcare-associated SARS-CoV-2 infection.

\section{Discussion}

We explored the effectiveness and safety of NIPPV in a cohort of COVID-19 patients admitted to non-ICU wards. Overall, $44 \%$ of patients responded to NIPPV, avoiding ETI and AHRF-related death. The rate of success was remarkably high (64\%) in the subgroup of patients deemed suitable for escalation to invasive MV. In contrast, a high percentage (76\%) of NIPPV failure and subsequent AHRF-related death was registered among older patients with a DNI order. Delivery of NIPPV to COVID-19 patients appeared safe for HCWs, as only 2 out of 124 individuals (1.6\%) experienced a SARS-CoV-2 infection while on activity in the COVID-19 wards, in the absence of serious symptoms.

In general, the use of NIPPV in patients with AHRF is expected to improve oxygenation, decrease the work of breathing, and avoid ETI, reducing the complications associated with invasive MV, such as pneumonia, excessive sedation, delirium, and ICU-acquired weakness [7]. Risks of NIPPV include large tidal volumes and injurious transpulmonary pressures and delayed initiation of invasive MV in
Table 3 Predictors of NIPPV success, univariate and multivariate analysis

\begin{tabular}{|c|c|c|c|c|}
\hline Variables & $\begin{array}{l}\text { Univariable OR } \\
(95 \% \mathrm{CI})\end{array}$ & $P$ value & $\begin{array}{l}\text { Multivariable OR } \\
(95 \% \mathrm{CI})\end{array}$ & $P$ value \\
\hline Age, years ${ }^{\mathbf{a}}$ & $0.94(0.89-1)$ & 0.041 & $1.11(0.95-1.28)$ & 0.183 \\
\hline Female sex (vs. male) & $0.58(0.17-1.94)$ & 0.375 & - & - \\
\hline Atrial fibrillation & $0.62(0.17-2.22)$ & 0.463 & & \\
\hline Cardiovascular disease & $0.57(0.18-1.79)$ & 0.337 & - & - \\
\hline Chronic kidney disease & $0.25(0.05-1.33)$ & 0.103 & - & - \\
\hline Cognitive impairment & $0.46(0.08-2.64)$ & 0.383 & - & - \\
\hline COPD & $0.81(0.2-3.34)$ & 0.776 & - & - \\
\hline Diabetes mellitus & $0.88(0.24-3.28)$ & 0.852 & - & - \\
\hline Hypertension & $0.9(0.29-2.77)$ & 0.854 & - & - \\
\hline Immunodeficiency & $2.7(0.23-31.89)$ & 0.430 & - & - \\
\hline Malignancy & $0.22(0.02-2.03)$ & 0.181 & - & - \\
\hline Stroke & $0.37(0.7-2.03)$ & 0.250 & - & - \\
\hline Total number of comorbidities ${ }^{\mathbf{a}}$ & $0.73(0.49-1.09)$ & 0.122 & - & - \\
\hline SOFA score sa $^{a}$ & $0.76(0.47-1.26)$ & 0.290 & - & - \\
\hline $\mathrm{PaO}_{2} / \mathrm{FiO}_{2}$ before NIPPV, $\mathrm{mm} \mathrm{Hg}^{\mathrm{a}}$ & $1(0.99-1.01)$ & 0.511 & - & - \\
\hline $\begin{array}{l}\mathrm{PaO}_{2} / \mathrm{FiO}_{2} 24-48 \mathrm{~h} \text { after NIPPV } \\
\text { initiation, } \mathrm{mm} \mathrm{Hg} \mathrm{Hg}^{\mathrm{a}}\end{array}$ & $1.01(1-1.03)$ & 0.008 & $1.02(1-1.03)$ & 0.015 \\
\hline NIPPV CPAP (vs. BiPAP) & $2.7(0.85-8.57)$ & 0.091 & - & - \\
\hline Antibiotics & $0.77(0.1-5.94)$ & 0.801 & - & - \\
\hline Corticosteroids & $4.75(1.14-19.83)$ & 0.033 & $15.4(1.79-132.57)$ & 0.013 \\
\hline Tocilizumab & $5.52(1.53-19.86)$ & 0.009 & $6.35(0.88-45.86)$ & 0.067 \\
\hline DNI order & $0.18(0.05-0.61)$ & 0.006 & $0.03(0.001-0.57)$ & 0.020 \\
\hline
\end{tabular}

${ }^{\text {aPer } 1-u n i t ~ i n c r e a s e ~}$ 
a rapidly decompensating patient, which can increase the risk of death and nosocomial spread of the infection [4, 13]. Previous studies on the use of NIPPV in patients with AHRF due to pandemic viral illnesses have yielded conflicting results, with failure rates ranging from 10 to $70 \%$ in patients with influenza, H1N1 and severe acute respiratory syndrome (SARS), and up to $92.4 \%$ in patients with middle east respiratory syndrome (MERS) [4, 7, 14]. Furthermore, NIPPV is an aerosol generating procedure (AGP) with the potential to increase the risk of SARS-CoV-2 infection transmission to HCWs, as shown in previous studies on the SARS epidemic $[4,11,15,16]$.

Despite controversies over the benefits and risks of NIPPV, reports from several countries have shown that $11-62 \%$ of patients hospitalized with severe to critical COVID-19 received NIPPV [17-21]. In Italy, NIPPV has been widely used, especially in non-ICU setting, since the huge number of patients with COVID-19-related AHRF outweighed the provision of ICU beds and ventilators [22, 23].

The results of our series seem to indicate that NIPPV, delivered via face mask in a non-ICU setting, is considerably effective in treating AHRF in COVID-19 patients with no limited life expectancy. In addition, the death rate of patients undergoing delayed ETI after an initial unsuccessful trial of NIPPV (33.3\%) did not exceed the mortality reported for ARDS from any cause (35-45\%) and death rates registered in series of mechanically ventilated COVID-19 patients [24]. This result, although obtained on a limited number of patients, seems to indicate that performing a delayed ETI in COVID-19 patients who failed an initial trial of NIPPV is not associated with a worse outcome than in patients who underwent early ETI and MV, differently from what some previous studies on ARDS suggest [13, 25].

The high mortality rate $(88 \%)$ registered in our series among patients with limited life expectancy indicate a scarce utility of NIPPV in this subset of COVID-19 individuals. However, if intubation and MV appear as an inappropriate choice, NIPPV could still play a role in the therapeutic management of such frail patients, since it allows, with a limited resource investment, to cure a small but not negligible proportion of patients (12\%) and deliver palliative care to dying subjects with COVID-19-related respiratory failure [7].

Regarding predictors of NIPPV success, the multivariable logistic regression model identified several factors independently associated with NIPPV outcome (Table 3). The analysis showed that an increase in the $\mathrm{PO}_{2} / \mathrm{FiO}_{2}$ ratio 24-48 $\mathrm{h}$ after NIPPV initiation was predictive of successful weaning: as a consequence, the results of blood gas analysis acquired the day after the start of NIPPV might be used to early identify potential responders to NIPPV and patients needing a treatment escalation toward ETI and ICU transfer.

Among the explored medical treatments, the use of corticosteroids was associated with a higher probability of
NIPPV success; this result appears in line with recent evidence demonstrating a survival benefit and a lower rate of progression towards invasive MV in hypoxemic COVID-19 patients receiving steroids [26].

Finally, the presence of a treatment limitation decision (DNI order), which was established based on a global clinical assessment of the patient, appeared as a strong predictor of NIPPV failure. This finding underlines the role of the clinician who, thanks to his/her experience, is able to evaluate the whole picture and early identify patients with poor prognosis whose primary goal is palliative care.

HCWs represent a category at high risk of COVID-19 infection: up to $3.8 \%$ (1.716 of 44.674 confirmed cases) of the reported cases in China and up to $12 \%$ (30.225 of 250.973) of all cases of COVID-19 in Italy have been among healthcare personnel $[27,28]$. These data highlight the crucial need to protect healthcare professionals, by adopting effective infection prevention and control measures.

SARS-CoV-2 is transmitted from person to person directly through respiratory droplets, or indirectly, via contaminated fomites. Another potential mode of transmission is the airborne route, i.e. the inhalation of respiratory particles smaller than droplets, generated by procedures such as noninvasive ventilation, ETI, tracheotomy, and manual ventilation before intubation (AGP) [8,9]. Based on these assumptions, airborne precautions (wearing of a respirator mask), in addition to droplet and contact precautions, are universally recommended when AGP are performed on COVID-19 patients [4, 9-11]. Moreover, international guidelines suggest performing AGP in negative pressure isolation rooms $[4,10,11]$. Previous studies have shown that the adoption of adequate airborne precautions, and the use of negative pressure systems can minimize the risk of infection among HCWs caring for SARS patients treated with NIPPV [15, 29]. At our institution, HCWs caring for COVID-19 patients treated with NIPPV were equipped with respirators, and full contact and droplet precautions, according to national and international guidelines [4, 9-11,30]. The activity at the COVID-19 general wards started about 2 weeks after the COVID-19 epidemic hit the regions of northern Italy. As a result, we had time to procure sufficient supply of PPE and to train staff on the proper use protective equipment. The low SARS-CoV-2 infection rate recorded among HCWs (1.6\%) confirms that the PPE adopted is highly effective in preventing coronavirus transmission during NIPPV delivery, even in the absence of negative pressure rooms or HEPA air filtering systems. We do not know if the use of NIPPV would be equally safe in settings where PPE are in short supply.

Some authors have recently advocated the use of helmet interface for NIPPV delivery in COVID-19 patients, in spite of face masks, as a better fitting and tolerable interface, which might minimize widespread dispersion 
of exhaled air and reduce the risk of airborne SARSCoV-2 transmission to HCWs [22, 23, 31, 32]. Furthermore, evidence exists that NIPPV delivered by helmet in patients with AHRF presents an advantage in terms of decreased intubation and improved mortality with respect to face mask [33, 34]. To date, however, no direct evidence of benefit of helmet over face mask in the treatment of COVID-19-associated AHRF exists [4]. At our institution, no helmet interfaces were available during the study period, and NIPPV was delivered exclusively via full-face or oronasal face masks. We attempted to limit SARS-CoV-2 spread into the ambient air by selecting nonvented masks and applying an antimicrobial filter to the exhalation port of the NIPPV circuits [35].

Our study presents several limitations. The limited number of enrolled patients and the retrospective design reduce the generalizability of our results. Due to the retrospective nature of the study, we were unable to retrieve detailed settings of NIPPV (e.g., PEEP, driving pressure, $\mathrm{FiO}_{2}$ ), respiratory rate and blood gas analysis parameters in the first hours after NIPPV initiation, and data on patient tolerance. The absence of a control group does not allow direct comparison of the cure rate between patients treated with NIPPV and patients undergoing early intubation.

Regarding the safety assessment of the use of NIPPV in COVID-19 patients, we did not compare the rate of SARSCoV-2 infection between HCWs caring for NIPPV-treated patients and HCWs caring for non-ventilated patients, since the entire healthcare staff cared for both patient subgroups. As a consequence, we do not know which is the specific contribution of NIPPV to nosocomial transmission of SARS-CoV-2.

\section{Conclusions}

Apart from elderly patients with limited life expectancy, NIPPV was effective in a substantially high percentage of patients with COVID-19-associated AHRF in this study. The rate of SARS-CoV-2 infection among HCWs caring for COVID-19 patients receiving NIPPV was quite low, suggesting that NIPPV is a safe practice, provided that strict adherence to appropriate infection prevention and control measures is ensured.

NIPPV could serve as an affordable and widely available supportive strategy for the treatment of patients with COVID-19-related AHRF admitted to non-ICU wards, especially in contexts of limited resources and limited ICU capacity, and/or in patients with "do not resuscitate/intubate" orders. Further research is needed to confirm our encouraging results.
Funding This research did not receive any specific grant from funding agencies in the public, commercial, or not-for-profit sectors.

\section{Compliance with ethical standards}

Conflict of interest The authors declare that they have no conflicts of interest.

Ethical standards The authors declare that all procedures performed in this study are in accordance with ethical standards of the institutional and national research committee and with the 1964 Helsinki declaration and its later amendments or comparable ethical standards.

\section{References}

1. Fan E, Beitler JR, Brochard L et al (2020) COVID-19-associated acute respiratory distress syndrome: is a different approach to management warranted? Lancet Respir Med 8(8):816-821. https ://doi.org/10.1016/S2213-2600(20)30304-0

2. Cummings MJ, Baldwin MR, Abrams D et al (2020) Epidemiology, clinical course, and outcomes of critically ill adults with COVID-19 in New York city: a prospective cohort study. Lancet 395(10239):1763-1770. https://doi.org/10.1016/S0140 $-6736(20) 31189-2$

3. World Health Organization. Clinical management of COVID19. Interim guidance 27 May 2020. WHO/2019-nCoV/ clinical/2020.5.

4. Alhazzani W, Møller MH, Arabi YM et al (2020) Surviving sepsis campaign: guidelines on the management of critically Ill adults with coronavirus disease 2019 (COVID-19). Crit Care Med 48(6):e440-e469. https://doi.org/10.1097/CCM.0000000000 004363

5. Frat JP, Thille AW, Mercat A et al (2015) High-flow oxygen through nasal cannula in acute hypoxemic respiratory failure. $\mathrm{N}$ Engl J Med 372(23):2185-2196. https://doi.org/10.1056/NEJMo a1503326

6. Bellani G, Laffey JG, Pham T et al (2017) Noninvasive ventilation of patients with acute respiratory distress syndrome insights from the LUNG SAFE study. Am J Respir Crit Care Med 195(1):67-77. https://doi.org/10.1164/rccm.201606-1306OC

7. Rochwerg B, Brochard L, Elliott MW et al (2017) Official ERS/ ATS clinical practice guidelines: noninvasive ventilation for acute respiratory failure. Eur Respir J 50(2):1602426. https://doi. org/10.1183/13993003.02426-2016

8. World Health Organization. Transmission of SARS-CoV-2: implications for infection prevention precautions. 9 July 2020. WHO reference number: WHO/2019-nCoV/Sci_Brief/ Transmission_modes/2020.3.

9. World Health Organization. Advice on the use of masks in the context of COVID-19. Interim guidance 5 June 2020. WHO reference number: WHO/2019-nCov/IPC_Masks/2020.4.

10. European Centre for Disease Prevention and Control. Infection prevention and control for COVID-19 in healthcare settings Fourth update. 3 July 2020. ECDC: Stockholm; 2020.

11. Lynch JB, Davitkov P, Anderson DJ et al (2020) Infectious diseases society of America guidelines on infection prevention for health care personnel caring for patients with suspected or known COVID-19. Clin Infect Dis. https://doi.org/10.1093/cid/ciaa1063

12. COVID-19 Treatment Guidelines Panel. Coronavirus Disease 2019 (COVID-19) Treatment Guidelines. National Institutes of Health. Available at https://www.covid19treatmentguidelines.nih. gov/. Accessed August 13, 2020. 
13. Chiumello D, Brochard L, Marini JJ et al (2017) Respiratory support in patients with acute respiratory distress syndrome: an expert opinion. Crit Care 21(1):240. https://doi.org/10.1186/s1305 4-017-1820-0

14. Alraddadi BM, Qushmaq I, Al-Hameed FM et al (2019) Noninvasive ventilation in critically ill patients with the Middle East respiratory syndrome. Influenza Other Respir Viruses 13:382-390

15. Fowler RA, Guest CB, Lapinsky SE et al (2004) Transmission of severe acute respiratory syndrome during intubation and mechanical ventilation. Am J Respir Crit Care Med 169(11):1198-1202. https://doi.org/10.1164/rccm.200305-715OC

16. Tran K, Cimon K, Severn M, Pessoa-Silva CL, Conly J (2012) Aerosol generating procedures and risk of transmission of acute respiratory infections to healthcare workers: a systematic review. PLoS ONE 7(4):e35797. https://doi.org/10.1371/journ al.pone. 0035797

17. Grasselli G, Zangrillo A, Zanella A et al (2020) Baseline characteristics and outcomes of 1591 patients infected with SARS$\mathrm{CoV}-2$ admitted to ICUs of the Lombardy region. Italy. JAMA 323(16):1574-1581. https://doi.org/10.1001/jama.2020.5394

18. Guan WJ, Ni ZY, Hu Y et al (2020) Clinical characteristics of coronavirus disease 2019 in China. N Engl J Med. https://doi. org/10.1056/NEJMoa2002032

19. Huang C, Wang Y, Li X et al (2020) Clinical features of patients infected with 2019 novel coronavirus in Wuhan, China. Lancet 395:497-506

20. Wang D, Hu B, Hu C et al (2020) Clinical characteristics of 138 hospitalized patients with 2019 Novel coronavirus-infected pneumonia in Wuhan. JAMA, China. https://doi.org/10.1001/ jama.2020.1585

21. Yang X, Yu Y, Xu J et al (2020) Clinical course and outcomes of critically ill patients with SARS-CoV-2 pneumonia in Wuhan, China: a single-centered, retrospective, observational study. Lancet Respir Med. https://doi.org/10.1016/S2213-2600(20)30079-5

22. Longhini F, Bruni A, Garofalo E et al (2020) Helmet continuous positive airway pressure and prone positioning: a proposal for an early management of COVID-19 patients. Pulmonology 26(4):186-191. https://doi.org/10.1016/j.pulmoe.2020.04.014

23. Lucchini A, Giani M, Isgrò S, Rona R, Foti G (2020) The "helmet bundle" in COVID-19 patients undergoing non invasive ventilation. Intensive Crit Care Nurs 58:102859. https://doi. org/10.1016/j.iccn.2020.102859

24. Quah P, Li A, Phua J (2020) Mortality rates of patients with COVID-19 in the intensive care unit: a systematic review of the emerging literature. Crit Care 24(1):285. https://doi.org/10.1186/ s13054-020-03006-1

25. Kangelaris KN, Ware LB, Wang CY et al (2016) Timing of intubation and clinical outcomes in adults with acute respiratory distress syndrome. Crit Care Med 44(1):120-129. https://doi.org/10.1097/ CCM.0000000000001359.PMID:26474112;PMCID:PMC47 74861
26. Recovery CG, Horby P, Lim WS et al (2020) Dexamethasone in hospitalized patients with Covid-19 - preliminary report. N Engl J Med. https://doi.org/10.1056/NEJMoa2021436

27. Wu Z, McGoogan JM (2020) Characteristics of and important lessons from the coronavirus disease 2019 (COVID-19) outbreak in China: summary of a report of 72314 cases from the Chinese center for disease control and prevention. JAMA. https://doi. org/10.1001/jama.2020.2648

28. Istituto Superiore di Sanita' (ISS). Epidemia COVID-19. Aggiornamento nazionale 11 agosto 2020.

29. Cheung TM, Yam LY, So LK et al (2004) Effectiveness of noninvasive positive pressure ventilation in the treatment of acute respiratory failure in severe acute respiratory syndrome. Chest 126(3):845-850. https://doi.org/10.1378/chest.126.3.845

30. Gruppo di lavoro ISS Prevenzione e controllo delle Infezioni. Indicazioni ad interim per un utilizzo razionale delle protezioni per infezione da SARS-COV-2 nelle attività sanitarie e sociosanitarie (assistenza a soggetti affetti da covid19) nell'attuale scenario emergenziale SARS-COV-2. Versione del 10 maggio 2020. Roma: Istituto Superiore di Sanità; 2020 (Rapporto ISS COVID-19, n. 2/ 2020 Rev. 2).

31. Hui DS, Chow BK, Lo T et al (2015) Exhaled air dispersion during noninvasive ventilation via helmets and a total facemask. Chest 147(5):1336-1343. https://doi.org/10.1378/chest.14-1934

32. Radovanovic D, Rizzi M, Pini S, Saad M, Chiumello DA, Santus P (2020) Helmet CPAP to treat acute hypoxemic respiratory failure in patients with COVID-19: a management strategy proposal. J Clin Med 9(4):1191. https://doi.org/10.3390/jcm9041191

33. Patel BK, Wolfe KS, Pohlman AS, Hall JB, Kress JP (2016) Effect of noninvasive ventilation delivered by helmet vs. face mask on the rate of endotracheal intubation in patients with acute respiratory distress syndrome: a randomized clinical trial. JAMA 315(22):2435-2441. https://doi.org/10.1001/jama.2016.6338

34. Ferreyro BL, Angriman F, Munshi L et al (2020) Association of noninvasive oxygenation strategies with all-cause mortality in adults with acute hypoxemic respiratory failure: a systematic review and meta-analysis. JAMA 324(1):1-12. https://doi. org/10.1001/jama.2020.9524

35. Pfeifer M, Ewig S, Voshaar T et al (2020) Position paper for the state-of-the-art application of respiratory support in patients with COVID-19. Respiration 99(6):521-542. https://doi. org/10.1159/000509104

Publisher's Note Springer Nature remains neutral with regard to jurisdictional claims in published maps and institutional affiliations. 